\title{
Low-dose chloroquine treatment extends the lifespan of aged rats
}

\section{Dear Editor,}

Chloroquine (CQ) has long been used as an anti-malarial agent (Wellems and Plowe, 2001). Recently, CQ has also been applied to treat viral infection and related diseases (Wellems and Plowe, 2001; Huang et al., 2020). However, the safety and efficacy of its applications are still under extensive debate (Solomon and Lee, 2009). Here, we discovered that low-dose $C Q$ has a geroprotective effect on physiologically aged rats. Low-dose CQ prolonged lifespan, repressed systemic inflammation, and inhibited fibrosis across multiple tissue types in aged rats. Furthermore, we constructed transcriptomic maps for 6 tissues (kidney, small intestine, liver, heart, lung, and aorta) upon CQ treatment, thus revealing the effects of $C Q$ at a systemic level. $C Q$ treatment mitigated age-related molecular changes and repressed genes linked to fibrosis and the inflammatory response. Altogether, our data provide a valuable resource for investigating the impact of $C Q$ on multiple aged tissues, which may facilitate the development of clinical applications that mitigate age-related changes in the elderly.

$C Q$ has been used to combat malaria for decades (Wellems and Plowe, 2001). As one kind of aminoquinoline drugs, CQ displays several advantages: easily absorbable, simple to synthesize, high efficacy, straightforward to use, and generally affordable (Solomon and Lee, 2009; Huang et al., 2020). CQ is used to treat a range of diseases, including inflammatory diseases, infectious diseases, and even multiple types of cancer (Solomon and Lee, 2009). CQ also alleviates the symptoms of rheumatoid arthritis, indicating its anti-inflammation property (Sargiacomo et al., 2020; Schrezenmeier and Dorner, 2020). In addition, studies increasingly indicate that $C Q$ treatment is involved in the regulation of cellular senescence (Sargiacomo et al., 2020). $C Q$ treatment results in a decreased ratio of cells that are positive for senescence-associated beta-galactosidase (SA$\beta$-gal) (Sargiacomo et al., 2020), a well-known senescent marker (Cai et al., 2020). However, whether CQ treatment attenuates systemic aging and extends the lifespan of naturally aged individuals is unclear.

Despite the beneficial effects of $C Q$ listed above, side effects have also been reported, eliciting widespread concerns. For example, high-dose $C Q$ increases the risk of cardiac arrhythmia with cardiotoxicity (Wozniacka et al., 2006). Side effects have also been observed in other tissues, such as gastrointestinal tissues and the liver (Solomon and Lee, 2009). Notably, these side effects depend on the dose, administration method, and the duration of treatment (Solomon and Lee, 2009). Therefore, it is essential to carefully evaluate the side effects of $C Q$ treatment to ensure safety at both tissue and organismal levels.

To evaluate the impact of CQ on senescence, we treated human mesenchymal stem cells (hMSCs), multipotent stem cells present in most tissues, with the drug. We first tested a wide range of concentrations from $0.2 \mu \mathrm{mol} / \mathrm{L}$ to $100 \mu \mathrm{mol} / \mathrm{L}$ of CQ in Werner syndrome-specific mesenchymal stem cells (WS hMSCs) (Fig. 1A), a stem cell model for premature aging (Wu et al., 2018). We found that CQ promoted cell selfrenewal at lower concentrations $(0.2-5 \mu \mathrm{mol} / \mathrm{L})$, but inhibited cell proliferation at higher concentrations $(20 \mu \mathrm{mol} / \mathrm{L}$ or above) (Fig. 1B). Specifically, treatment with $1 \mu \mathrm{mol} / \mathrm{L} \mathrm{CQ}$ decreased the percentage of SA- $\beta$-gal-positive cells and increased the number of Ki67-positive cells in WS hMSCs (Fig. 1C and 1D). Additionally, low-dose CQ treatment decreased IL-6 secretion of WS hMSCs (Fig. 1E). Our previous work indicated that the loss of heterochromatin was associated with the senescence of WS hMSCs (Wu et al., 2018; Bi et al., 2020), and we found low-dose CQ treatment upregulated the levels of heterochromatin-associated marks including $\mathrm{H} 3 \mathrm{~K} 9 \mathrm{me} 3, \mathrm{HP} 1 \mathrm{Y}$, and LAP2, indicating that $\mathrm{CQ}$ treatment restored the heterochromatin landscape in WS hMSCs (Fig. S1A). Collectively, our results suggest that lowdose $C Q$ alleviates senescence in prematurely aged stem cells.

To further examine the systemic effects of $C Q$ in vivo, we treated 24-month-old Sprague Dawley (SD) male rats with $\mathrm{CQ}$ twice a week for 5 months at a low dose of $0.1 \mathrm{mg} / \mathrm{kg}$ orally by water to avoid potential side effects (Fig. 1A). Lowdose $C Q$ administration extended the lifespan of rats by approximately $6 \%$ in terms of median longevity and by about $13 \%$ in terms of maximum longevity (Fig. 1F). CQ-treated rats also tended to have decreased serum TNF- $\alpha$ levels and reduced the numbers of circulating white blood cells (WBC) and neutrophils (NEU) in old rats (Figs. 1G-J and S1B), suggestive of attenuated chronic inflammation. In addition, CQ-treated rats maintained comparable body weights and 


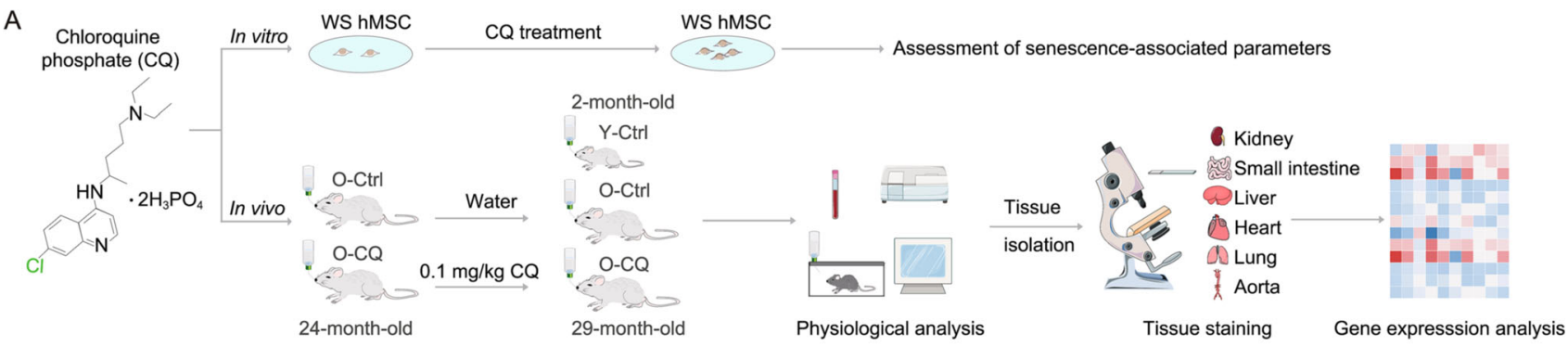

B

WS hMSC

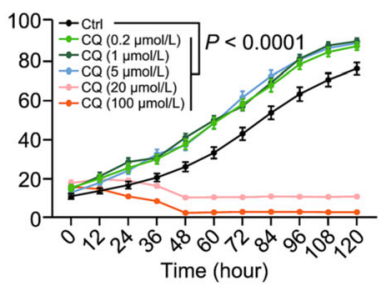

C

ws hMSC

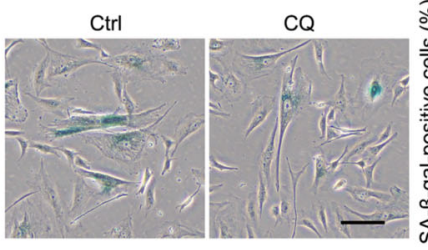

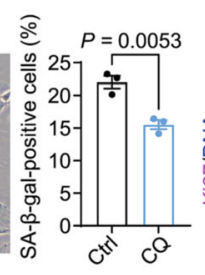

D

ws hMSC
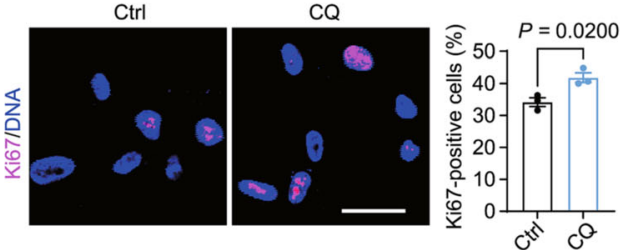

E

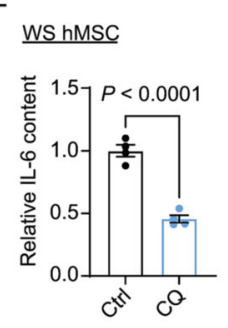

F

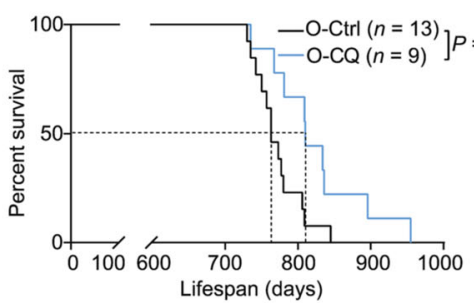

K
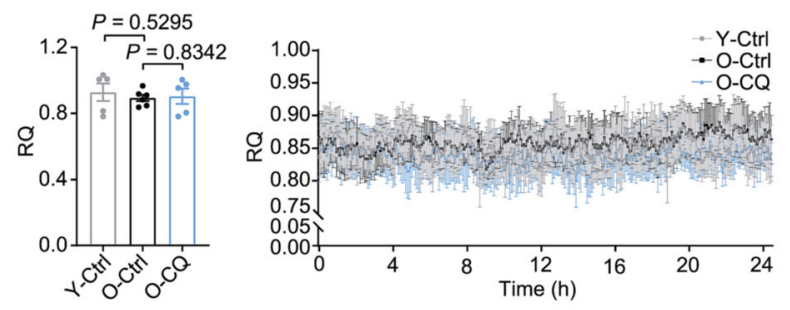

M
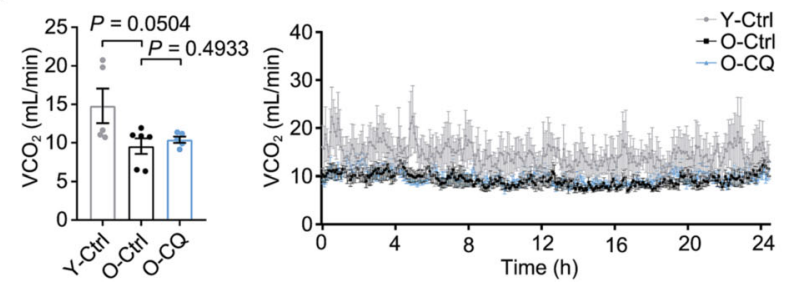

G

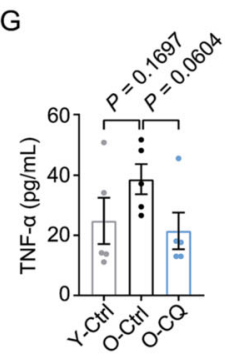

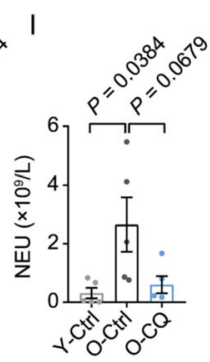

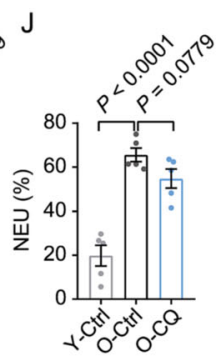

L
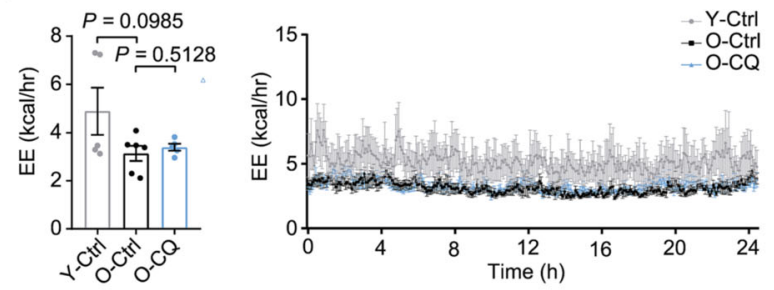

N

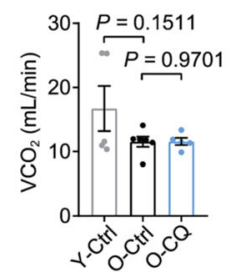

$\rightarrow$ Y-Ctrl

$\rightarrow \mathrm{O}-\mathrm{Ctrl}$
$-\mathrm{CQ}$

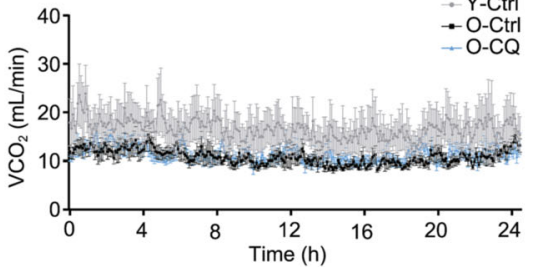


4 Figure 1. The geroprotective effect of $\mathrm{CQ}$ on cellular senescence and tissue aging. (A) Schematic diagram showing analysis of the $\mathrm{CQ}$ effectiveness in vitro and in vivo. Upper, the determination of an optimal concentration of $\mathrm{CQ}$ for a geroprotective role in WS hMSCs. Lower, the treatment of 24-month-old rats with drinking water (O-Ctrl) or CQ (O-CQ) for five months. Y-Ctrl, 2-month-old rats with drinking water. (B) Relative cell proliferation analysis upon $C Q$ treatment at given concentrations in WS hMSCs (passage 7) by using the IncuCyte S3 live cell imaging system. The data are shown as means \pm SD. $n=6$ biological replicates per group. (C) SA- $\beta$-gal staining of CQ-treated and control WS hMSCs (passage 7). Scale bar, $100 \mu \mathrm{m}$. The data are shown as means \pm SEM. $n=3$ biological replicates per group (unpaired Student's $t$-test). (D) Immunostaining of Ki67 in CQ-treated and control WS hMSCs (passage 7). Scale bar, $25 \mu \mathrm{m}$. Data are shown as means \pm SEM. $n=3$ biological replicates per group (unpaired Student's $t$-test). (E) ELISA of IL- 6 in CQ-treated and control WS hMSCs (passage 7). The data are shown as means \pm SEM. $n=4$ per group (unpaired Student's $t$-test). (F) Survival curves for O-Ctrl ( $n=13$ rats) and O-CQ $(n=9$ rats) rats (Log-rank test). (G) ELISA of TNF- $\alpha$ in the plasma of Y-Ctrl, O-Ctrl and $\mathrm{O}-\mathrm{CQ}$ rats. The data are shown as means \pm SEM. $n=5$ rats per group (unpaired Student's $t$-test). $(H)$ The number of white blood cells (WBC) in the blood. The data are shown as means \pm SEM. $n=5$ rats per group (unpaired Student's $t$-test). (I) The number of neutrophils (NEU) in the blood. The data are shown as means \pm SEM. $n=5$ rats per group (unpaired Student's $t$-test). (J) The proportion of neutrophils (NEU) in the blood. The data are shown as means \pm SEM. $n=5$ rats per group (unpaired Student's $t$-test). (K) Real-time measurement of respiratory quotient (RQ) of Y-Ctrl, O-Ctrl and O-CQ rats by metabolic cage detection. Right, representative curves of $R Q$ measured within $24 \mathrm{~h}$. The data are shown as means \pm SEM. $n=5-6$ rats per group (unpaired Student's $t$-test). (L) Real-time measurement of energy expenditure (EE, kcal/h) of Y-Ctrl, O-Ctrl and O-CQ rats by metabolic cage detection. Right, representative curves of EE measured within $24 \mathrm{~h}$. The data are shown as means \pm SEM. $n=5-6$ rats per group (unpaired Student's $t$-test). (M) Real-time measurement of carbon dioxide emission rate $\left(\mathrm{VCO}_{2}, \mathrm{~mL} / \mathrm{min}\right)$ in $\mathrm{Y}-\mathrm{Ctrl}, \mathrm{O}-\mathrm{C}$ trl and $\mathrm{O}-\mathrm{CQ}$ rats by metabolic cage detection. Right, representative curves of carbon dioxide emission measured within $24 \mathrm{~h}$. The data are shown as means \pm SEM. $n=5-$ 6 rats per group (unpaired Student's $t$-test). (N) Real-time measurement of oxygen consumption rate $\left(\mathrm{VO}_{2}, \mathrm{~mL} / \mathrm{min}\right)$ of Y-Ctrl, O-Ctrl and O-CQ rats by metabolic cage detection. Right, representative curves of oxygen consumption measured within 24 h. The data are shown as means \pm SEM. $n=5-6$ rats per group (unpaired Student's $t$-test).

blood glucose levels to age-matched control rats (Fig. S1C). Metabolic cage analysis was used to track the metabolic activity of CQ-treated rats. Respiratory quotient, energy expenditure, carbon dioxide emission rate and oxygen consumption rate were largely unaffected by $C Q$ treatment (Figs. $1 \mathrm{~K}-\mathrm{N}$ ). Altogether, our results demonstrate that lowdose $C Q$ extends rat lifespan without affecting standard physiological metrics reflecting metabolic health.

To evaluate the effects of $C Q$ on different tissues, we examined the effect of $C Q$ in the kidney, liver, heart, small intestine, and lung, all of which are key organs that contribute to systemic aging. Generally, the tissue index (tissue weight divided by body weight) and morphological integrity were unaffected by $C Q$ treatment despite the fact that the absolute weight of liver was decreased by $C Q$ treatment (Figs. S1D, S1E and S2A). We inspected aging-related parameters in tissues using histological examination, fibrosis evaluation, aggresome staining and SA- $\beta$-gal staining (Geng et al., 2019). CQ treatment alleviated fibrosis in multiple aged tissues, such as kidney, liver, and lung (Fig. 2A). In addition, the number of CD68-positive macrophages and aggresome-positive cells in the liver (Figs. S2B and S2C), as well as levels of alanine aminotransferase (ALT) and total bilirubin (TBiL) in the blood were reduced after $C Q$ treatment (Fig. 2B), suggesting that $C Q$ has a liver-protective effect. However, CQ failed to reduce the levels of $S A-\beta$-gal positivity in most tissues, albeit with a decreasing trend in lung (Fig. S2D). Notably, the cardiac function seems not adversely affected by low dose of $C Q$ treatment in aged rats (Fig. 2C). Collectively, these data suggest that the low-dose $\mathrm{CQ}$ treatment alleviates aging phenotypes in a tissuespecific manner without detectable cardiac side effects.

To uncover the effects of $C Q$ on tissue-specific gene expression, kidney, small intestine, liver, heart, lung, and aorta tissues were collected from CQ-treated and control groups and subjected to high-throughput RNA sequencing along with 2-month-old rats as young controls (Fig. 2D). By evaluating the transcriptomes from young and old control rats, we identified aging-related differentially expressed genes "Aging DEGs" across multiple tissues (Figs. S3A and $\mathrm{S} 3 \mathrm{~B})$. By comparing transcriptomes from $C Q$-treated and control old rats, we defined CQ-induced differentially expressed genes "CQ DEGs" across multiple tissues (Figs. S3A and S3B). Further exploration of the potential effects of $C Q$ on aging-associated gene sets identified four groups of DEGs with distinct biological implications, i) aging $D E G$ s that were reversed by $C Q$ treatment, labeled "Rescue DEGs", ii) aging DEGs that were unchanged upon $C Q$ treatment, called "Failure to rescue DEGs", iii) CQ-DEGs that were unchanged during aging, namely " $C Q$ specific DEGs", and iv) aging DEGs that were exacerbated after $C Q$ treatment, namely "Pro-aging DEGs" (Fig. S3A). Notably, tissue specificity was observed in the number and distribution of these four types of DEGs, suggesting that $C Q$ exhibits a tissue-dependent effect (Fig. S3B). Taken together, we established the transcriptomic landscape of multiple tissues in response to aging and $C Q$ treatment in rats, which 

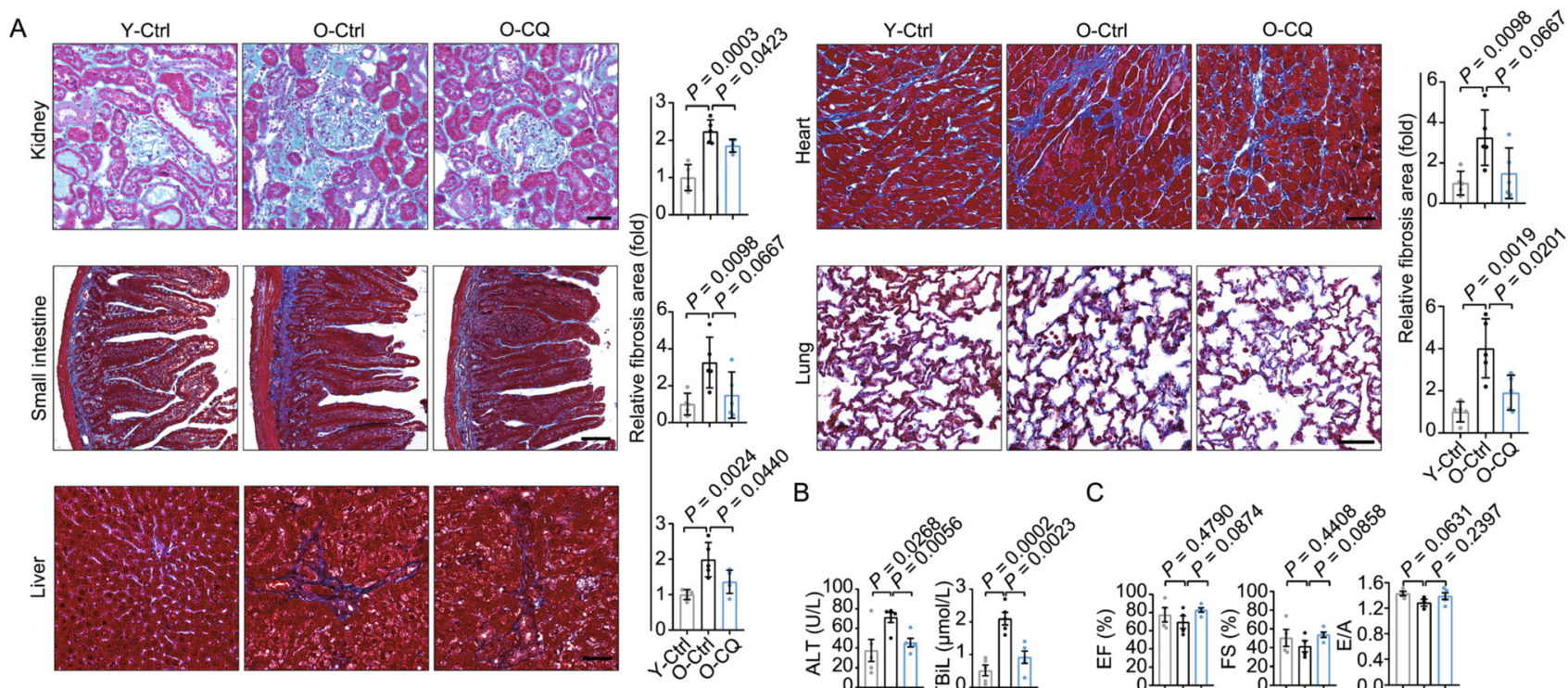

B
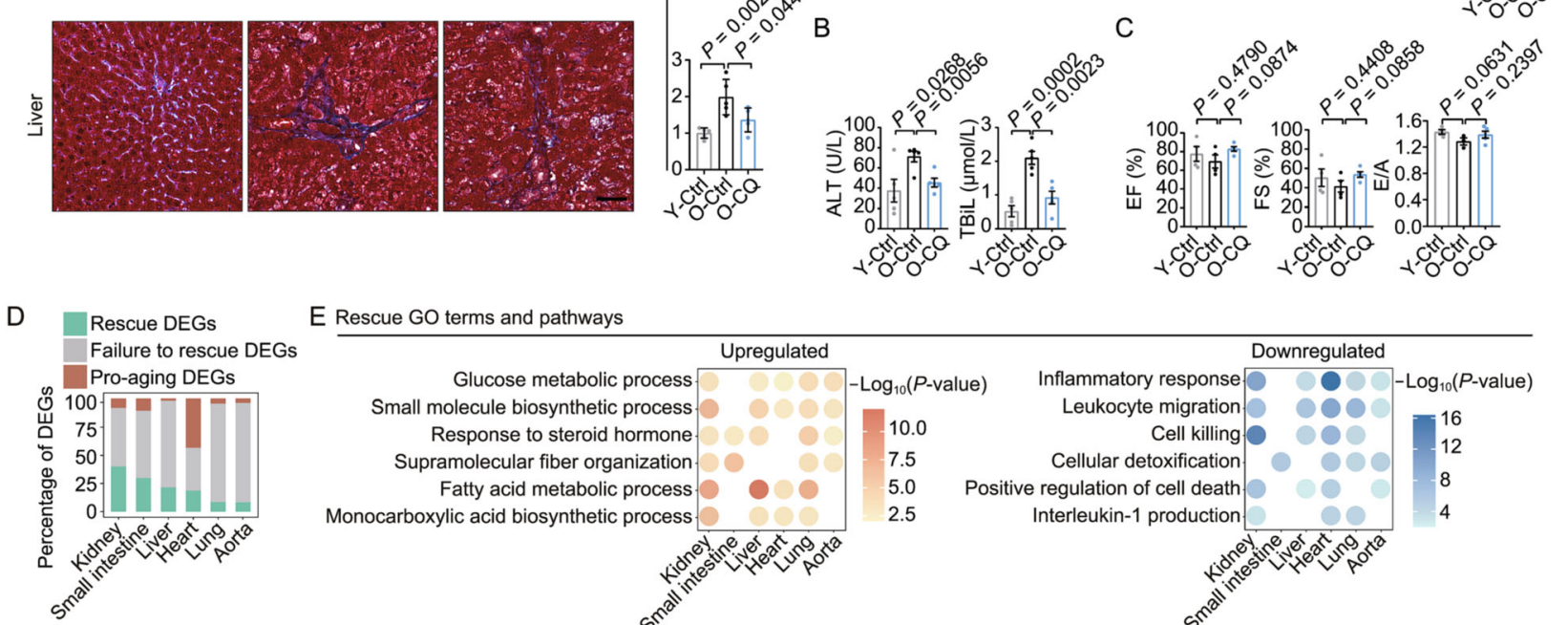

E Rescue GO terms and pathways

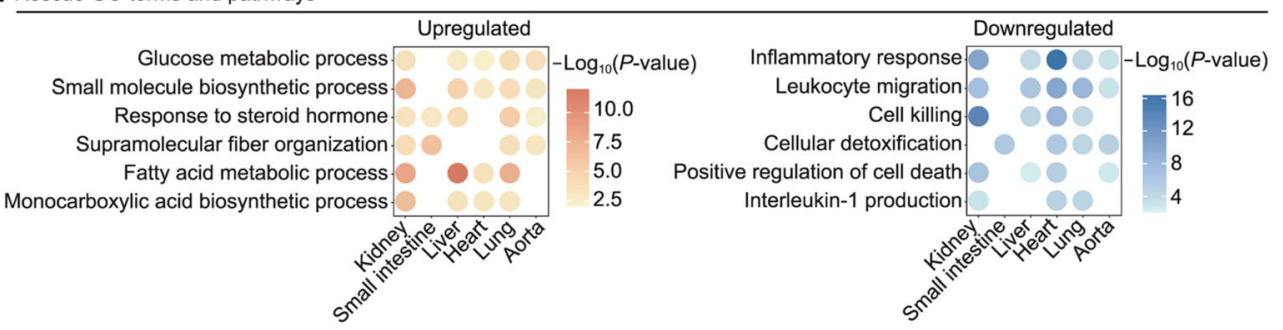

$\mathrm{F}$

Inflammatory response
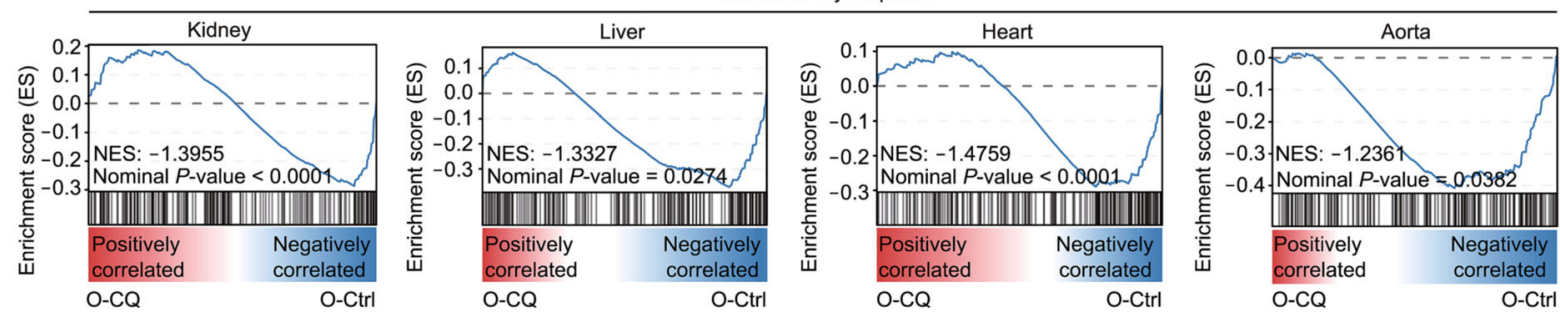

G Overlap between rescue DEGs and genes of kidney disease databases

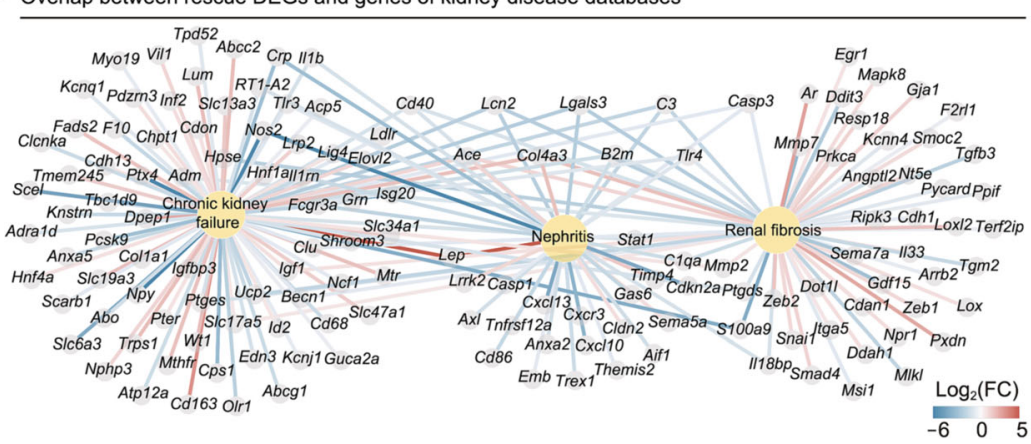

$\mathrm{H}$

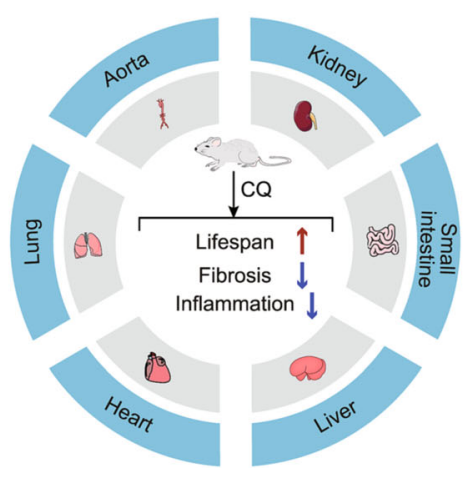


4 Figure 2. Changes in the transcriptional profiles of different tissues during aging and $C Q$ treatment. (A) Masson staining of kidney, small intestine, liver, heart and lung, tissues from Y-Ctrl, O-Ctrl, O-CQ-treated rats. Scale bars, $60 \mu \mathrm{m}$ for lung, $50 \mu \mathrm{m}$ for liver and heart and $100 \mu \mathrm{m}$ for kidney and small intestine. The data are shown as means \pm SEM. $n=5$ rats per group (unpaired Student's $t$-test). (B) Alanine aminotransferase (ALT) and total bilirubin (TBiL) detection in the plasma. The data are shown as means \pm SEM. $n=5$ rats per group (unpaired Student's $t$-test). (C) Left ventricular ejection fraction (EF), left ventricular fractional shortening (FS), and the ratio of peak velocity of early to late filling of mitral inflow (E/A) of Y-Ctrl, $\mathrm{O}-\mathrm{Ctrl}$ and $\mathrm{O}-\mathrm{CQ}$ rats. The data are shown as means \pm SEM. $n=3-5$ rats per group (unpaired Student's $t$-test). (D) Bar plot showing the percentages of rescue DEGs, failure to rescue DEGs and pro-aging DEGs in the six tissues. Green indicates rescue DEGs, gray indicates a failure to rescue DEGs, and brown indicates pro-aging DEGs. (E) Representative GO terms and pathways enriched in rescue DEGs shared by at least three tissues. (F) Gene set enrichment analysis (GSEA) plots showing decreased inflammatory response in kidney, liver, heart and aorta upon CQ treatment. (G) Network plot showing rescue DEGs associated with renal diseases in kidney. (H) Schematic diagram illustrating the geroprotective effects of $C Q$ in different tissues.

enables us to delineate the potential effects of $C Q$ on aging in a tissue-specific manner.

Consistent with the observation that $C Q$ treatment alleviated the signs of tissue aging and extended the lifespan of aged rats, $C Q$ treatment rescued the expression of aging DEGs in tissues to varying extents (Fig. 2D). For instance, about $40 \%$ of genes in aged kidneys, $30 \%$ of genes in aged small intestines, and $20 \%$ of genes in aged livers were rescued by $C Q$ treatment (Figs. $2 D$ and S3A). These results suggest that $C Q$ treatment mitigates aging-related molecular changes in a tissue-specific manner. The most significant dynamic changes in gene expression were observed in kidney, consistent with the phenotypic improvement described above (Fig. 2A). We systematically analyzed the "Rescue DEGs" using Gene Ontology (GO) terms across six tissues to uncover any common effects of $C Q$ across multiple tissues. We found that the genes in the categories of glucose metabolic process, small molecule biosynthetic process, fatty acid metabolic process, and monocarboxylic acid biosynthetic process were upregulated in at least four tissues upon $C Q$ treatment, and were reduced during aging (Fig. 2E). By contrast, genes associated with the inflammatory response, leukocyte migration, cell killing, and positive regulation of cell death, were increased during aging and were downregulated in at least four tissues upon $C Q$ administration (Fig. 2E). Additionally, gene set enrichment analysis (GSEA) confirmed that CQ treatment attenuated the gene expression involved in the inflammatory response, IFN response, and senescence-associated secretory phenotype (SASP) in multiple aged tissues (Figs. 2F, S3C and S3D). IL6-JAK-STAT3 and pro-fibrotic TGF- $\beta$ signaling pathways were also downregulated by $\mathrm{CQ}$ treatment in aged livers (Figs. S3E and S3F).

We also performed an integrative comparative analysis of our transcription profiles with aging/longevity-associated genes from the Aging Atlas database (Aging Atlas, 2021). We found that the expression of aging-associated genes, such as Cdkn2b, Cdkn2a, and Cdkn1a were downregulated by $C Q$ treatment (Fig. S3G). To unravel the systemic effects of $C Q$ treatment, we further focused on those 76 "Rescue DEGs" that were shared by at least three tissues (Fig. S4A). Among these genes, the pro-inflammatory factors S100a9, S100a8, Cxcl1, and II1b were decreased, and Hsp90aa1, a major molecular chaperon that maintains protein homeostasis and thereby plays a cytoprotective role, was increased in multiple tissues after $C Q$ treatment (Fig. S4A). These results suggest that low-dose $C Q$ treatment stimulates the upregulation of a systemic geroprotective transcriptional network across different tissues.

Our analysis revealed that the kidney showed the upregulation of the most rescue genes upon $\mathrm{CQ}$ treatment (Fig. 2D). This explains the prominent geroprotective effects of $\mathrm{CQ}$ treatment on kidney (Figs. 2D and S3B), indicating that low-dose $C Q$ improves nephron functions, including metabolic processing of nutrients and removal of waste materials (Fig. S4B). In addition, GO terms such as response to virus, leukocyte mediated cytotoxicity, and immune effector process were repressed by $C Q$ in aged kidneys (Fig. S4B). Consistently, rescue DEGs upregulated by $\mathrm{CQ}$ contained a series of kidney-specific organic anion transporters, such as S/c17a2, S/c24a3, S/c31a1 and S/c36a4 (Fig. S4C), which play vital roles in regulating anion balance in the body. Furthermore, CQ treatment attenuated both CXCR-associated chemotaxis genes, including Cxcl1, Cxcl2, Cxcl6, and $\mathrm{Cxc} / 9$ and genes harboring interferon-stimulated responsive element (ISRE), such as $M \times 1, M \times 2$, Oas1a, Oas2, and Cgas (Figs. S4D and S4E). Additionally, we verified that upregulation of $M \times 2$, an IFN-stimulated gene that mediates innate immunity, was inhibited by $C Q$ treatment in aged kidney through RT-qPCR (Fig. S4F). We also performed an integrative comparison analysis of our transcription profiles with genes associated with kidney diseases and found that $C Q$-associated rescue DEGs correlated with the onset of a series of kidney diseases, such as renal fibrosis, nephritis, and chronic kidney failure (Fig. 2G). Thus, our data suggest that low-dose $C Q$ treatment exhibits a beneficial effect in aged kidneys, potentially reducing innate immune response and improving ion transport function. Consistent with the beneficial effect of $C Q$ on kidney, we found that $C Q$ treatment downregulated genes associated with neutrophil chemotaxis, leukocyte migration and macrophage activation, while upregulated genes associated with fatty acid metabolic process, small molecule catabolic process and cellular catabolic process in aged liver (Fig. S4G), 
further indicating the potential hepato-protective effects of $\mathrm{CQ}$.

Furthermore, we investigated the biological implications of pro-aging genes that were altered by $C Q$ treatment. More than half of the pro-aging DEGs $(1,678 / 2,934)$ were detected in the heart, most of which $(1,594 / 1,678)$ were upregulated (Fig. S3A). By integrative analysis of pro-aging DEGs with genes associated with heart diseases, we found that $C Q$ treatment-induced gene networks were associated with hypertrophic cardiomyopathy, cardiac arrhythmia, and heart failure (Fig. S4H). For instance, Caps2, which is also increased in patients with refractory ischemic end-stage heart failure, was further augmented by $C Q$ treatment in aged heart (Fig. S4I). In addition, Myh7, a gene associated with cardiomyopathy, was upregulated in aged heart and further augmented by $\mathrm{CQ}$ (Fig. S4H). These data imply that despite seemingly normal heart function (Fig. 2C), low-dose $\mathrm{CQ}$ treatment may be associated with pro-aging side effects at the transcriptional level.

In this study, we found that low concentrations of $\mathrm{CQ}$ alleviated stem cell senescence, repressed tissue fibrosis, and extended lifespan (Fig. 2H). Multi-tissue transcriptomic inspection demonstrated that $C Q$ may have both beneficial and detrimental effects on aged animals in a tissue-specific manner. By surveying the transcriptomic landscape of $C Q$ treated tissues, we found that low-dose $\mathrm{CQ}$ treatment attenuated age-associated gene expression across tissues. The strongest effect was observed in the kidney where we found decreased levels of ISRE-containing genes and increased expression of transporter encoding genes. However, $C Q$ also augmented pro-aging transcriptional signatures, which may elicit potential cardiac toxicity without detectable functional impairment during the duration of the experiment. Our work systemically evaluates the phenotypic and transcriptional effects of $\mathrm{CQ}$ across mammalian tissues, thus providing a valuable resource to interrogate the potential effects of low-dose CQ usage.

Our transcriptomic profiling demonstrated the impact of $\mathrm{CQ}$ on 6 tissues in aged rats, and revealed tissue-specific effects of $C Q$. We identified aged kidneys as the most efficiently rescued tissue in terms of rescue DEGs. CQ treatment attenuated chronic inflammation and ISRE-containing genes, such as OAS/RNaseL, IFN, and cGAS. In addition, low-dose $C Q$ treatment upregulated the genes involved in systemic ion homeostasis in the kidney and could at least partly rescue the transcriptomic signatures of multiple kidney diseases. Consistent with our results, $C Q$ decreases renal fibrosis in chronic kidney diseases by inhibiting the lysosomal protease cathepsin D (Fox et al., 2016). However, CQ also had adverse effects on aged hearts at the transcriptional level. Most pro-aging DEGs were enriched in the heart, with an overrepresentation of cardiac disease-related gene sets. Consistently, the clinical cardiovascular toxicity of $\mathrm{CQ}$ has been reported (Wozniacka et al., 2006). For example, the treatment with chloroquine or hydroxychloroquine decreases myocardial cell discharge, leading to aberrant cardiac electrical conduction and a prolonged corrected QT interval based on electrocardiography (Wozniacka et al., 2006; Solomon and Lee, 2009). By contrast, although $C Q$ has noticeable effects in hearts at the transcriptional level, extremely low-dose $C Q$ may not affect normal heart function as assessed by transthoracic echocardiography and overall physiological health.

Notably, we found that low-dose $C Q$ treatment extended the lifespan of naturally aged rats. The dose of CQ used in this study was only $0.1 \mathrm{mg} / \mathrm{kg}$ twice a week, at least 100 -fold lower than the dose that was previously used in rodents (Solomon and Lee, 2009). Our results support the potential efficacy of $\mathrm{CQ}$ in delaying aging. Furthermore, it has been reported that $3.5 \mathrm{mg} / \mathrm{kg}$ of $\mathrm{CQ}$ enhanced DNA damage clearance and rescued age-related metabolic shift, suggesting a potential geroprotective effect (Qian et al., 2018). This lifespan extension effect by $C Q$ was also observed recently in nematodes and a progeria mouse model (Qian et al., 2018). Our study shows that low-dose CQ extended the lifespan of naturally aged rodents.

The role of $C Q$ in counteracting aging may be linked to its ability to inhibit chronic inflammation systematically and alleviate fibrosis. Consistent with our observations, CQ reduces inflammation and effectively decreases the salivary and serum levels of IL-6, a key component of SASP (Sargiacomo et al., 2020). In addition, CQ treatment is beneficial in alleviating mouse liver fibrosis induced by carbon tetrachloride $\left(\mathrm{CCl}_{4}\right)$ via the inhibition of autophagy pathways (He et al., 2014). In paraquat-induced lung injury, CQ regulates oxidative stress, attenuates lung inflammation, and reduces fibrosis (Shen et al., 2017). Collectively, our data, together with others, indicate that $C Q$ has beneficial roles in reducing chronic inflammation and tissue fibrosis, which could be harnessed to treat different age-related diseases.

In summary, our results demonstrate a geroprotective role of low-dose CQ on prematurely senescent human stem cells and physiologically aged rats. In general, these findings enable us to gain an in-depth understanding of the potential impact of $C Q$ on organ aging and further emphasize the necessity of monitoring cardiac function when using $C Q$ in clinical trials.

\section{FOOTNOTES}

The authors are grateful to Jingyi Jia (Xuanwu Hospital Capital Medical University), Xiang Shi, Xinyi Wu, Sai Yang, Jiajia Hou, Zhuanzhuan Xing, and Zheng Liu (Laboratory Animal Center, Institute of Biophysics, CAS) for the management of laboratory animals, Lei Bai, Qun Chu, Xiao Zhuo, Jing Lu, Ying Yang, Ruijun Bai, Luyang Tian and Shikun Ma for administrative assistance. This work was supported by the National Key Research and Development Program of China (2018YFC2000100), the Strategic Priority Research Program of the Chinese Academy of Sciences (XDA16000000), the Program of Beijing Municipal Science and Technology Commission (Z191100001519005), the National Natural Science Foundation of China (Grant Nos. 81921006, 81625009, 
91749202, 81861168034, 91949209, 92049304, 81822018, 81870228, 81922027, 82071588, 92049116, 31801010, 81901433, 82125011, 82122024, 92149301, 92168201), the National Key Research and Development Program of China (2020YFA0804000, 2020YFA0112200, 2017YFA0103304, 2017YFA0102802, 2018YFA0107203, 2020YFA0113400), the Program of the Beijing Natural Science Foundation (Z190019, JQ20031), the Key Research Program of the Chinese Academy of Sciences (KFZDSW-221), K. C. Wong Education Foundation (GJTD-2019-06, GJTD2019-08), Beijing Hospitals Authority Youth Programme (QML20200802), Youth Innovation Promotion Association of CAS (E1CAZW0401, 2021078), the Non-profit Central Research Institute Fund of Chinese Academy of Medical Sciences (2020-JKCS-011), the State Key Laboratory of Stem Cell and Reproductive Biology, the State Key Laboratory of Membrane Biology, the 14th Five-year Network Security and Informatization Plan of Chinese Academy of Sciences (WX145XQ07-18), the Informatization Plan of Chinese Academy of Sciences (CAS-WX2021SF-0301), the Milky Way Research Foundation (MWRF), Young Elite Scientists Sponsorship Program by CAST (NO.YESS20200012), CAS Project for Young Scientists in Basic Research (YSBR-012) and the Tencent Foundation.

The authors declare no conflict of interest.

Wei $\mathrm{Li}^{1,8}$, Zhiran Zou ${ }^{2,6,7,9}$, Yusheng Cai ${ }^{2,6,9}$,

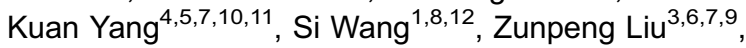
Lingling Geng ${ }^{1,8}$, Qun Chu ${ }^{3,6,9}$, Zhejun $\mathrm{Ji}^{3,6,9}$, Piu Chan ${ }^{8}$, Guang-Hui Liu ${ }^{1,2,6,7,8,9 凶}$, Moshi Song ${ }^{2,6,7,9 凶}$, Jing Qu ${ }^{3,6,7,9 凶}$,' Weiqi Zhang $4,5,6,7,8,10 \bowtie]$

${ }^{1}$ Advanced Innovation Center for Human Brain Protection, National Clinical Research Center for Geriatric Disorders, Xuanwu Hospital Capital Medical University, Beijing 100053, China

2 State Key Laboratory of Membrane Biology, Institute of Zoology, Chinese Academy of Sciences, Beijing 100101, China

3 State Key Laboratory of Stem Cell and Reproductive Biology, Institute of Zoology, Chinese Academy of Sciences, Beijing 100101, China

${ }^{4}$ CAS Key Laboratory of Genomic and Precision Medicine, Beijing Institute of Genomics, Chinese Academy of Sciences, Beijing 100101, China

${ }^{5}$ China National Center for Bioinformation, Beijing 100101, China

${ }^{6}$ Institute for Stem Cell and Regeneration, Chinese Academy of Sciences, Beijing 100101, China

7 University of Chinese Academy of Sciences, Beijing 100049, China

${ }^{8}$ Aging Translational Medicine Center, Xuanwu Hospital, Capital Medical University, Beijing 100053, China

${ }^{9}$ Beijing Institute for Stem Cell and Regenerative Medicine, Beijing 100101, China

${ }^{10}$ Sino-Danish College, University of Chinese Academy of Sciences, Beijing 101408, China

11 Sino-Danish Center for Education and Research, Beijing 101408, China
12 Chongqing Renji Hospital, University of Chinese Academy of Sciences, Chongqing 400062, China

$\bowtie$ Correspondence: ghliu@ioz.ac.cn (G.-H. Liu), songmoshi@ioz. ac.cn (M. Song), qujing@ioz.ac.cn (J. Qu), zhangwq@big.ac.cn (W. Zhang)

\section{OPEN ACCESS}

This article is licensed under a Creative Commons Attribution 4.0 International License, which permits use, sharing, adaptation, distribution and reproduction in any medium or format, as long as you give appropriate credit to the original author(s) and the source, provide a link to the Creative Commons licence, and indicate if changes were made. The images or other third party material in this article are included in the article's Creative Commons licence, unless indicated otherwise in a credit line to the material. If material is not included in the article's Creative Commons licence and your intended use is not permitted by statutory regulation or exceeds the permitted use, you will need to obtain permission directly from the copyright holder. To view a copy of this licence, visit http:// creativecommons.org/licenses/by/4.0/.

\section{REFERENCES}

Aging Atlas C (2021) Aging Atlas: a multi-omics database for aging biology. Nucleic Acids Res 49:D825-D830

Bi S, Liu Z, Wu Z, Wang Z, Liu X, Wang S, Ren J, Yao Y, Zhang W, Song $M$ et al (2020) SIRT7 antagonizes human stem cell aging as a heterochromatin stabilizer. Protein Cell 11:483-504

Cai Y, Zhou H, Zhu Y, Sun Q, Ji Y, Xue A, Wang Y, Chen W, Yu X, Wang $L$ et al (2020) Elimination of senescent cells by betagalactosidase-targeted prodrug attenuates inflammation and restores physical function in aged mice. Cell Res 30:574-589

Fox C, Cocchiaro P, Oakley F, Howarth R, Callaghan K, Leslie J, Luli S, Wood KM, Genovese F, Sheerin NS et al (2016) Inhibition of lysosomal protease cathepsin $D$ reduces renal fibrosis in murine chronic kidney disease. Sci Rep 6:20101

Geng L, Liu Z, Zhang W, Li W, Wu Z, Wang W, Ren R, Su Y, Wang P, Sun $L$ et al (2019) Chemical screen identifies a geroprotective role of quercetin in premature aging. Protein Cell 10:417-435

He W, Wang B, Yang J, Zhuang Y, Wang L, Huang X, Chen J (2014) Chloroquine Improved Carbon Tetrachloride-Induced Liver Fibrosis through Its Inhibition of the Activation of Hepatic Stellate Cells: Role of Autophagy. Biol Pharm Bull 37:1505-1509

Huang M, Li M, Xiao F, Pang P, Liang J, Tang T, Liu S, Chen B, Shu J, You $Y$ et al (2020) Preliminary evidence from a multicenter prospective observational study of the safety and efficacy of chloroquine for the treatment of COVID-19. Natl Sci Rev 7:14281436

Qian, M., Liu, Z., Peng, L., Tang, X., Meng, F., Ao, Y., Zhou, M., Wang, M., Cao, X., Qin, B., et al. (2018). Boosting ATM activity alleviates aging and extends lifespan in a mouse model of progeria. eLife 7.

Sargiacomo C, Sotgia F, Lisanti MP (2020) COVID-19 and chronological aging: senolytics and other anti-aging drugs for the 
treatment or prevention of corona virus infection. Aging 12:65116517

Schrezenmeier E, Dorner T (2020) Mechanisms of action of hydroxychloroquine and chloroquine: implications for rheumatology. Nat Rev Rheumatol 16:155-166

Shen H, Wu N, Wang Y, Zhao H, Zhang L, Li T, Zhao M (2017) Chloroquine attenuates paraquat-induced lung injury in mice by altering inflammation, oxidative stress and fibrosis. Int Immunopharmacol 46:16-22

Solomon VR, Lee H (2009) Chloroquine and its analogs: a new promise of an old drug for effective and safe cancer therapies. Eur J Pharmacol 625:220-233
Wellems TE, Plowe CV (2001) Chloroquine-resistant malaria. J Infect Dis 184:770-776

Wozniacka A, Cygankiewicz I, Chudzik M, Sysa-Jedrzejowska A, Wranicz JK (2006) The cardiac safety of chloroquine phosphate treatment in patients with systemic lupus erythematosus: the influence on arrhythmia, heart rate variability and repolarization parameters. Lupus 15:521-525

Wu Z, Zhang W, Song M, Wang W, Wei G, Li W, Lei J, Huang Y, Sang $Y$, Chan $P$ et al (2018) Differential stem cell aging kinetics in Hutchinson-Gilford progeria syndrome and Werner syndrome. Protein Cell 9:333-350
Wei Li, Zhiran Zou, Yusheng Cai, Kuan Yang and Si Wang have contributed equally.

Supplementary Information The online version contains supplementary material available at https://doi.org/10.1007/s13238-02100903-1. 